\title{
Single Electron Interference and Diffraction Experiments with a High Energy Physics Detector
}

\author{
Gian Luigi Alberghi ${ }^{* a}$ R. Doná ${ }^{a, b}$, A. Gabrielli ${ }^{a, b}$, F. Giorgi ${ }^{a, b}$, G. Matteucci ${ }^{b}$, \\ N. Semprini Cesari ${ }^{a, b}$, M. Villa ${ }^{a, b}, \mathbf{A}$. Zoccoli ${ }^{a, b}$, S. Frabboni ${ }^{c, d}$, G. Gazzadi ${ }^{d}$ \\ ${ }^{a}$ I.N.F.N. Bologna, I-40126 Bologna, Italy, \\ ${ }^{b}$ Department of Physics and Astronomy, Bologna University, I-40126 Bologna, Italy, \\ ${ }^{c}$ Department of Physics, University of Modena and Reggio Emilia, Via G. Campi 213/a, 41125 \\ Modena, Italy, \\ ${ }^{d}$ CNR-Institute of Nanoscience-S3, Via G. Campi 213/a, 41125 Modena, Italy \\ E-mail: alberghi@bo.infn.it
}

The Young-Feynman two-slit experiment for single electrons was carried out by inserting two nanometric slits and a fast recording system, capable of detecting the electron arrival-time, in a conventional transmission electron microscope. The sensor, designed for experiments at future high energy colliders, is based on a custom 4096 Monolithic Active Pixels CMOS chip, is equipped with a fast readout chain and can manage up to $10^{6}$ frames per second, allowing the collection of high statistic samples of single electron events within a time interval compatible with the stability of the experimental setup and the coherence conditions of the illumination. For the first time in a single electron two-slit experiment, the time distribution of electron arrivals was obtained.

11th International Conference on Large Scale Applications and Radiation Hardness of Semiconductor Detectors

3-5 July 2013

Auditorium Cassa di Risparmio di Firenze, via Folco Portinari 5, Florence, Italy

\footnotetext{
* Speaker.
} 


\section{Introduction}

The single electron two-slit interference is one of the most effective experiments in order to investigate the wave behaviour of material particles. This arrangement, adopted by Bohr and Einstein to develop their considerations on quantum mechanics foundations, is usually quoted as the Young-Feynman set-up. Up to now, the superposition of electron waves has been demonstrated in a variety of arrangements: single slit, single hole, double slit, double hole, multiple slits and electrostatic biprism (for a review on electron interferometry see [1]). Nevertheless, the most striking part of the experiment, i.e. the build-up of the interference pattern by the single electrons arriving on the final screen, has been observed only by means of very poor statistic samples [1].

The reason for this relies on the rate limitation of the available recording systems, such as cathode ray tube screens, able to collect at most just few tens of pictures per second, typically fifty (corresponding to the refresh rate), in conjunction with the time of stable operativity of the optical bench (i.e. an electron microscope), that rarely goes beyond few minutes. Mechanical vibrations, minimal variations in the objective excitation current are just few of the causes that leads to image drift and blur effects that degrades the fringe pattern. A trade-off between the number of electrons collected in a single frame and the amount statistics in the final pattern had to be found.

In this setup, a modern digital recording system, sensitive to single electrons, replaces the final viewing screen of the microscope, allowing to build-up a high statistics interference pattern (about $10^{5} e^{-}$) made in the vast majority of single-electron frames (more than 98\%), all within the time lapse of a few minutes, where the stable operativity and the coherence conditions of the microscope are guaranteed. Moreover, a large fraction of empty frames could be collected between the arrivals of two consecutive electrons, making it possible, for the first time in this kind of experiment, to measure the time distribution of electron arrival (see i.e. [2]).

Our detector is based on a custom CMOS chip of 4096 monolithic active pixels (MAPS), designed by the SLIM5 collaboration as a prototype for a vertex detector for experiments in future high energy colliders, and equipped with a fast read-out chain able to manage up to $10^{6}$ frames per second (fps) $[3,4]$.

In order to keep the experiment in the closest form to the original Young-Feynman set-up, the interference pattern was produced in the microscope by a gold-carbon film with couples of adjacent nano-slits prepared by using modern nanotechnology tools, rather than using an electrostatic biprism.

\section{The experimental apparatus}

\subsection{The slits}

The two nanometric slits were fabricated by a Focused Ion Beam (FIB) digging a golden layer about $250 \mathrm{~nm}$ thick, deposited by flash evaporation on a commercial copper grid, coated with a carbon film. The FIB milling was performed with a dual beam apparatus (FEI Strata DB235M), that combines a $30 \mathrm{keV}-\mathrm{Ga}^{+}$FIB with a thermal field emission scanning electron microscope (SEM), with a spatial resolutions of $6 \mathrm{~nm}$ and $2 \mathrm{~nm}$, respectively [5]. In particular, the slits we used were produced with a $10 \mathrm{pA}$ beam, corresponding to an ion beam spot-size of about $10 \mathrm{~nm}$. The beam was scanned over $100 \times 1500 \mathrm{~nm}$ rectangular patterns, $450 \mathrm{~nm}$ spaced, $5 \mathrm{~s}$ for each pattern. 

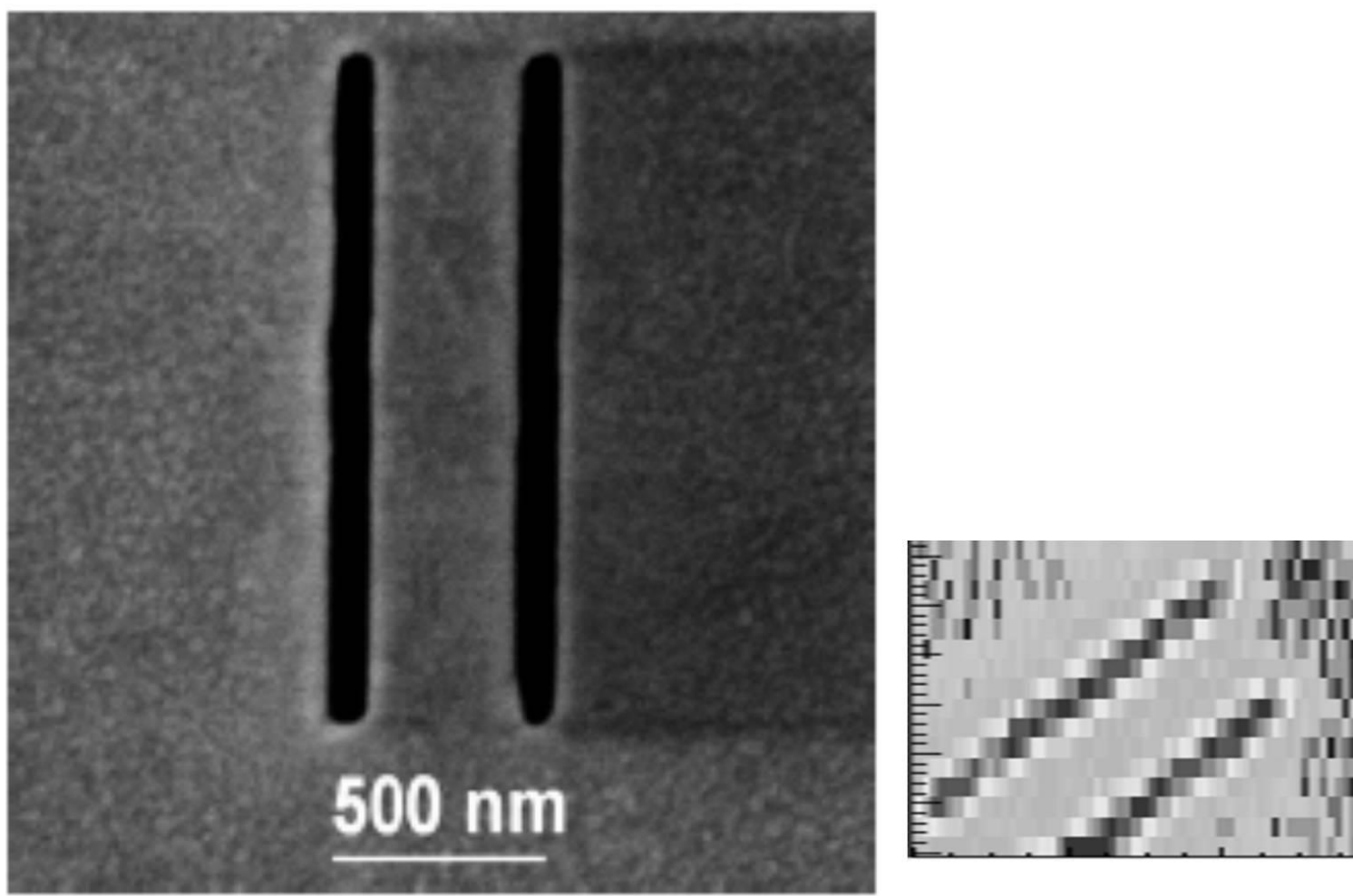

Figure 1: Slits images obtained with a scanning electron microscope (left) and with our experimental apparatus (right).

The passage through the gold-carbon bilayer was monitored by detecting the change in brightness of the ion-induced secondary electron emission. The slit width, length and spacing are $95 \mathrm{~nm}, 1550$ $\mathrm{nm}$ and $440 \mathrm{~nm}$ respectively, a SEM scan image is shown in figure 1.

\subsection{The electonic transmission microscope}

The interference experiments were carried out with a Philips EM400T transmission electron microscope (TEM), (see fig, 2), equipped with a tungsten hair-pin filament source operating at 60 $\mathrm{keV}$ with a corresponding de Broglie wavelength $\lambda$ pf $4.9 \mathrm{pm}$. Due to the small electron diffraction angles (of the order of $10^{-5} \mathrm{rad}$ ) associated with the separation of the slits and the wavelongth of the electrons, the Fraunhofer pattern must be observed in the so called low-angle diffraction mode. In this electro-optical set-up the condenser lenses were excited at their maximum strength, in order to reach the necessary lateral coherence of the illumination and the objective lens is weakly excited in order to project the Fraunhofer image onto the selected area aperture plane. In these conditions the microscope works as a diffraction camera having camera lengths extending up to several hundred meters. A picture of the overall apparatus is shown in figure 2.

The electron microscope working conditions, especially the current intensity, have been chosen in order to satisfy two conflicting requirements. On one hand it must be high enough to collect high statistics samples within a time interval that guarantees a stable operativity of the microscope (few minutes). On the other hand the current is strongly reduced to obtain the necessary coherence of the electron source Once the optimal working conditions of the electron microscope are 


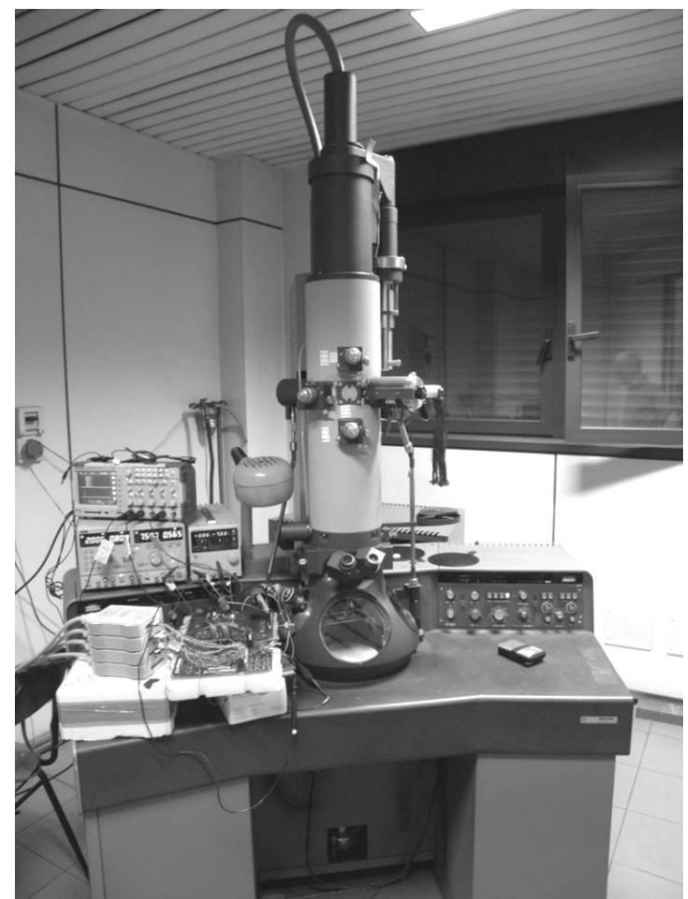

Figure 2: The experimental apparatus: the transmission electron microscope (Philips EM400T) and data acquisition system set up. The MAPS detector is installed inside the vacuum observation chamber. A high vacuum connector, mounted on a steel flange replacing the left window, provided the input/output signals and powering to the pixel sensor.

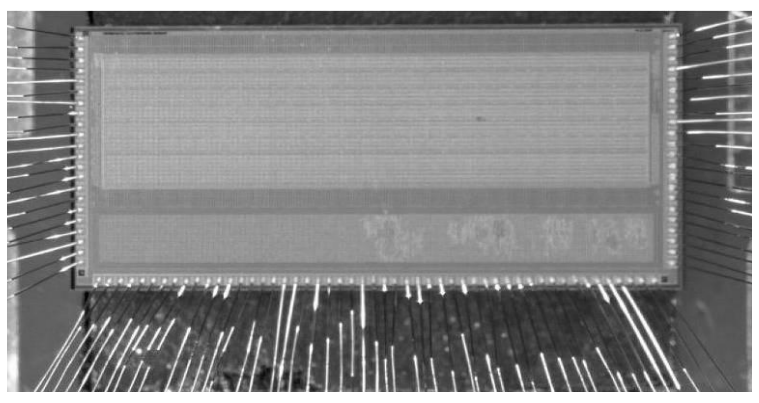

Figure 3: Picture of the monolithic CMOS pixel sensor Apsel4D, developed by the SLIM5 INFN Collaboration [4].

established, the acquisition frame rate has been chosen in order to contain, at the percent level, the fraction of frames presenting more than one single electron. Concerning the specific data-set presented in this paper, the multiple-electrons frames are about $1.5 \%$ of the single-electron frames.

\subsection{The sensor}

The sensor used for detecting electrons is a custom silicon chip named Apsel4D (figure 3). It is composed of a matrix of $128 \times 32$ active pixels developed in $0.13 \mu \mathrm{m}$ CMOS technology with a MAPS analog architecture and a fast digital sparsified readout [4]. Each square pixel has a 50 $\mu \mathrm{m}$ pitch and can provide a hit/not-hit information that is tagged with a time-stamp label. This 
sensor chip has been designed for the vertex detector's innermost layers for the next generation of particle physics experiments. It was designed in order to achieve high efficiency, high reliability and high particle rates (up to $100 \mathrm{MHz} / \mathrm{cm}^{2}$ ) by using low budget material. In order to satisfy the high efficiency requirement with high particle rate, a very fast and efficient ASIC digital readout was developed [6]. To keep high the readout speed the pixels have a fully digital design, i.e. they do not provide information on the detected charge. During functioning an external periodic signal, called BCO (Bunch Cross-Over) for historical reasons, is used to tag the time hit pixels. At each BCO signal, the matrix is swept in search for hit pixels that are read out from the matrix at a rate of 4 columns ( 32 pixel each) every 5 internal clock cycles. Read pixels become immediately sensitive to external signals thus minimizing the dead time.

Outside the matrix area, a sparsifier and buffering logic identify the hit pixels, providing the space and time association to hits and sending out the data at a rate of 1 hit per clock cycle. The proper use of the chip foresees that, on average, only a few pixels are hit during a BCO period, and only those hit pixels are actually sent out to a DAQ system. This is in contrast with a standard rolling-shutter imaging system where all pixels (having an amplitude information) are actually read and sent out.

The actual reading frequency and time-stamping capabilities depend on the read clock and the BCO clock frequency. The chip has been designed with peak performances of a $100 \mathrm{MHz}$ read clock and a $10 \mathrm{MHz}$ BCO clock frequencies. It was studied extensively on proton and pion beam tests at CERN [4], where spatial resolutions compatible with $50 / \sqrt{12} \mu \mathrm{m}$ have been observed. Runs performed with $1 \mathrm{MHz}$ BCO clock frequencies have demonstrated the capability of the chip to continuously stand a frame rate of $10^{6} \mathrm{fps}$. However, in the experimental conditions of the present data taking, a much more conservative $\mathrm{BCO}$ of about $6 \mathrm{kHz}$ has been used. Due to the low source brightness, the aforementioned frame rate was enough for the single-electron condition and, furthermore, made it possible to acquire a large set of empty frames spacing apart the electron events. This allowed the reconstruction of the difference time-of-arrival distribution with a $165 \mu \mathrm{s}$ resolution.

The data acquisition system, designed and realized for this particular experience, is based on an online hit monitoring and chip management setup. The digital output of the sensor goes through an electronic board designed for voltage level conversion and signal improvement to a Field Programmable Gate Array which decodes the information on the hit position and timing, redirecting the decrypted data to a personal computer.

The working conditions of the presented experiment, where a low electron flux is required, are quite relaxed for this high energy physics sensor so that the digital readout expected efficiency is almost $100 \%$.

\section{Results}

The data presented here were taken with a relatively long acquisition run (we achieved a reasonable stability of the system for about 20 minutes), collecting 96 thousands electrons. The average electron flux impinging on the sensitive surface of the detector was measured in the number of $80 e^{-} / \mathrm{s}$, corresponding to one electron hit every $12.5 \mathrm{~ms}$. Provided that the relativistic $\beta$ factor for a $60 \mathrm{keV}$ electron is about 0.45 , comparing the average time distance between the detected 
electrons to the time of flight within the electronic microscope (about $10 \mathrm{~ns}$ ), we see that almost every electron was already on disk before the next was emitted by the tungsten wire.

The off-line data analysis showed that $98.64 \%$ of the collected frames are empty, $1.34 \%$ contain single electron events, and $0.02 \%$ present two hits or more. The electron multiplicity in the collected frames is plotted in figure 4 (left). From the measurement of the time interval which separates two adjacent non-empty frames, we obtain the distribution of the differential electron time-of-arrival on the detector. The exponential behaviour expected in the case of uncorrelated electrons can be observed in figure 4 (right).
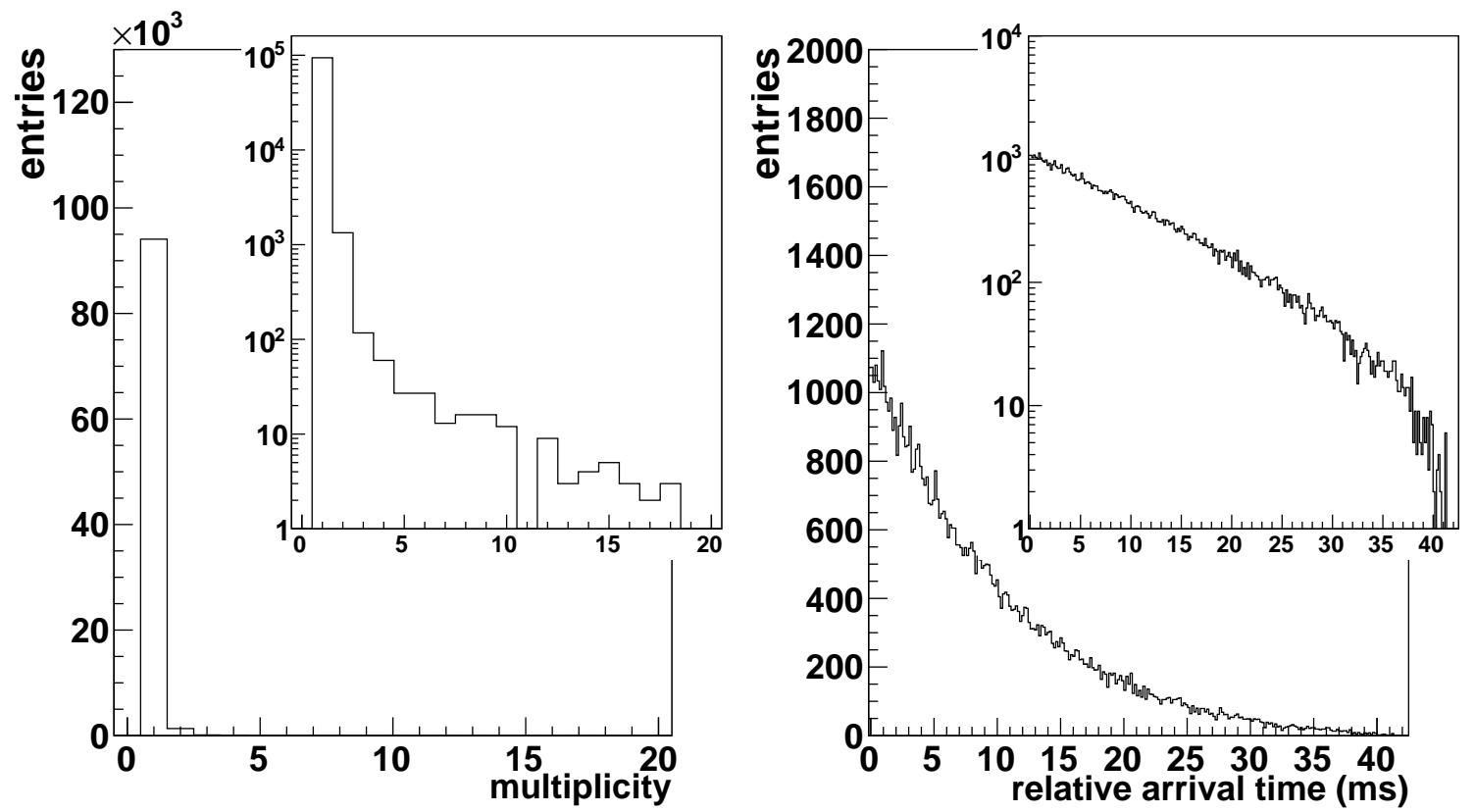

Figure 4: Multiplicity distribution of hit pixels in the frames (left). The bin zero, which is not plotted and counts the empty frames, is of the order of $7 \times 10^{6}$. Distribution of time intervals between two consecutive non-empty events (right).

The off-line frame integration process for the image reconstruction is sketched out in figure 5 . All the recorded frames were processed by the data-analysis program, integrating the hits on a $2 \mathrm{D}$ histogram. The post-processed data are plotted with a grey-scale code, revealing the interference pattern.

The coherence relation among the single electrons emerges when the different frames are added-up to form a single image. The interference scatter plot appears as expected from the interference of the two slits, placed at a certain distance, modulated by the diffraction due to their widths. In figure 6 we show the typical two-slit interference pattern that we obtained integrating the single electron frames.

\section{Conclusions}

We have realized for the first time the two-slit Young-Feynman experiment in its closest form to the original proposal. The time distribution and the build-up of high statistics interference pattern of the single electrons arriving on the screen were obtained for the first time. These results 


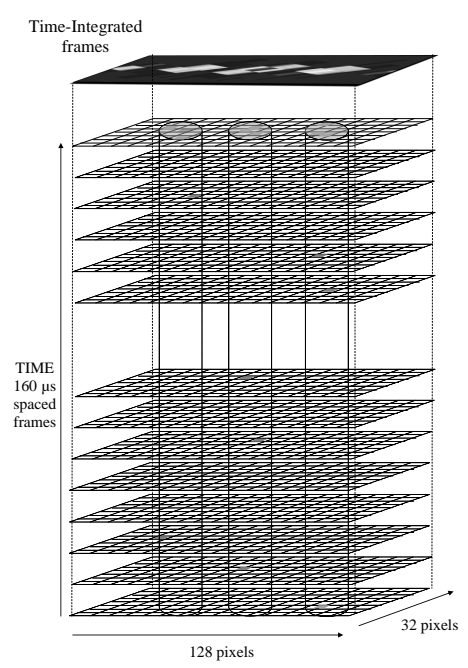

Figure 5: Pictorial view of the stack of frames collected in a typical run. Each frame is collected in $165 \mu \mathrm{s}$. Empty/Hit frame ratio not in scale

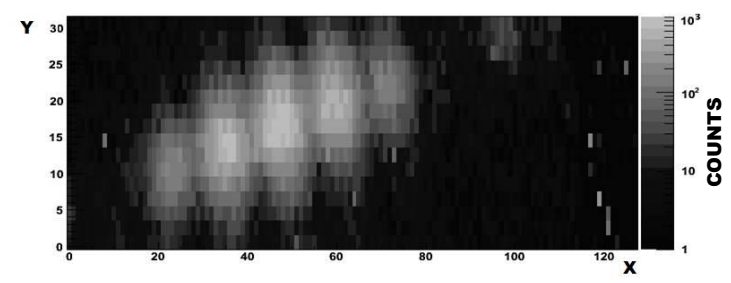

Figure 6: Interference pattern obtained adding-up the stack of frames.

open several and interesting perspectives for future developments. The high statistics of single electrons makes it possible to study the detailed properties of interference pattern formation which are connected to important tests of quantum mechanics. The system developed has an excellent potential in the field of electron microscopy, especially in the investigation of rare phenomena both in static and time-dependent [7] regimes.

\section{Acknowledgements}

We express our gratitude to Slim5 Collaboration and INFN Bologna Section for their technical support, in particular we thank G. Balbi, R. Berti, A. Costa and M. Lolli.

\section{References}

[1] F. Hasselbach., Progress in electron- and ion-interferometry, Rep. Prog. Phys. 73 (2010) 1

[2] G. Matteucci et al., Build-up of interference patterns with single electrons, Eur. J. Phys. 34 (2013) 511

[3] G. Rizzo et al., The SuperB silicon vertex tracker, http://dx.doi.org/10.1016/j.nima.2009.10.051 Nucl. Instr. Meth. A 617 (2011) 585 
[4] S. Bettarini et al., The SLIM5 low mas silicon tracker demonstrator, http://dx.doi.org/10.1016/j.nima.2010.08.026 Nucl. Instr. Meth. A 623 (2008) 942

[5] S. Frabboni, G. C. Gazzadi, G. Pozzi, Nanofabrication and the realization of Feynman's two-slit experiment, http://apl.aip.org/resource/1/applab/v93/i7/p073108_s1 Appl. Phys. Lett. 93 (2010) 7

[6] A. Gabrielli et al., A 4096-pixel MAPS device with on-chip data sparsification, http://dx.doi.org/10.1016/j.nima.2009.01.175 Nucl. Instr. Meth. A 604 (2009) 408

[7] A. H. Zewail, Four-dimensional electron microscopy, http://dx.doi.org/10.1126/science.1166135 Science 3285975 (2010) 187

[8] H. Batelaan, Colloquium, Illuminating the Kapitza-Dirac effect with electron matter optics, http://dx.doi.org/10.1103/RevModPhys.79.929 Rev. Mod. Phys. 793 (2007) 929 